\title{
AN UNSCENTED KALMAN FILTER FOR FREEWAY TRAFFIC ESTIMATION
}

\author{
L. Mihaylova*, R. Boel ${ }^{* *}$ and A. Hegyi ${ }^{* * *}$ \\ * Lancaster University, UK, mila.mihaylova@ieee.org \\ ** University of Ghent, Belgium,rene.boel@ugent.be \\ *** Delft University of Technology, the Netherlands, \\ a.hegyi@dcsc.tudelft.nl
}

\begin{abstract}
This paper addresses the problem of freeway traffic flow estimation. The freeway is considered as a network of components representing different freeway stretches called segments. The evolution of the traffic in a segment is modelled as a dynamic stochastic system, influenced by states of neighbour segments. Measurements are received only at boundaries between some segments and averaged within regular time intervals. An Unscented Kalman filter is developed and its performance is compared with a particle filter both for synthetic data and for real traffic data. The intended application is to supply traffic control systems with the estimated traffic state. Copyright@2006 IFAC
\end{abstract}

Keywords: macroscopic traffic models, unscented Kalman filter, particle filter

\section{INTRODUCTION}

Dynamic traffic control offers possibilities to avoid traffic jams on freeways by making better use of the available infrastructure. These control systems rely on measures such as ramp metering, dynamic speed limits and route guidance. The choice for the control actions is based on the current traffic state. However, the traffic state is typically unavailable everywhere and always on the traffic network, because of malfunctioning of sensors (magnetic loops or cameras) or because the sensors are sparsely installed. Further problems are that the measurements are corrupted by noise and that the control systems may need information about the traffic state that is not directly measured (e.g., density is generally not measured). In this paper these problems are tackled by using an Unscented Kalman Filter (UKF) which combines the knowledge about the traffic behaviour (i.e., a model) and the measurements.

The highly nonlinear behaviour of traffic can be described by macroscopic models (Hoogendoorn and Bovy 2001, Papageorgiou and Blosseville 1989, Helbing 2002) that are suitable for real-time problems in view of the fact that they represent the average traffic behaviour through aggregated variables (flow, density and speed at different locations). Most papers dealing with recursive traffic state estimation apply the Extended Kalman filter (EKF) to such macroscopic models. For example, (Wang and Papageorgiou 2005) propose an EKF to estimate the unknown parameters and states of a stochastic version of METANET macroscopic model (Papageorgiou and Blosseville 1989) of freeway traffic. These estimators have all the advantages and disadvantages of the EKF technique: presumably computationally cheap, but relying on a linearisation of the state and measurement models which can cause filter divergence.

In (Sun et al. 2003) a solution to highway traffic estimation is proposed by a sequential Monte Carlo algorithm, the so-called mixture Kalman filtering, and in (Mihaylova and Boel 2004) a particle filter $(\mathrm{PF})$ is developed. The mixture 
Kalman filter (MKF) (Chen and Liu 2000) is essentially a bank of Kalman filters run with the Monte Carlo sampling approach. The MKF is applicable only to conditionally linear Gaussian models. First-order traffic models represent the network, i.e. only the traffic density is modelled, distinguishing between the free-flow mode and congestion mode. The traffic mode is characterised in (Sun et al. 2003) by its density.

In contrast to (Sun et al. 2003), the traffic in the present paper is described by a second-order macroscopic model, and we develop an UKF that estimates both the density and speed. The traffic is described by the recently developed model (Boel and Mihaylova 2006) that is an extension to the cell-transmission model (Daganzo 1994). We compare the UKF to the PF from (Mihaylova and Boel 2004). The freeway network is modelled as a sequence of segments (Fig. 1). Sensors are available only at some boundaries between segments. Technological limitations (such as limited bandwidth of communication channels) force one to average these measurements over regular or irregular time intervals before they are transmitted to the centre where the measurement update is carried out.

The outline of the paper is as follows. Section 2 presents the stochastic macroscopic traffic model and the model for real-time traffic measurements used in the UKF. Section 3 describes the UKF for traffic estimation. The UKF performance is evaluated in Section 4. Conclusions and future research issues are highlighted in Section 5.

\section{FREEWAY TRAFFIC FLOW MODEL}

\subsection{Compositional Macroscopic Traffic Model}

Traffic states are estimated consecutively at discrete time instants $t_{1}, t_{2}, \ldots, t_{k}, \ldots$, based on the incoming information transmitted by sensors to the estimation algorithm. The overall state vector $\boldsymbol{x}_{k}=\left(\boldsymbol{x}_{1, k}^{T}, \boldsymbol{x}_{2, k}^{T}, \ldots, \boldsymbol{x}_{n, k}^{T}\right)^{T}$ at time $t_{k}$ consists of local state vectors $\boldsymbol{x}_{i, k}=\left(N_{i, k}, v_{i, k}\right)^{T}$, where $N_{i, k}$, [veh], is the number of vehicles counted in segment $i \in \mathcal{I}=\{1,2, \ldots n\}$, and $v_{i, k},[\mathrm{~km} / \mathrm{h}]$, is their average speed. The traffic state evolution is described by the system of equations

$$
\begin{aligned}
& \boldsymbol{x}_{1, k+1}=f_{1}\left(Q_{k}^{i n}, v_{k}^{\text {in }}, \boldsymbol{x}_{1, k}, \boldsymbol{x}_{2, k}, \boldsymbol{\eta}_{1, k}\right), \\
& \boldsymbol{x}_{i, k+1}=f_{i}\left(\boldsymbol{x}_{i-1, k}, \boldsymbol{x}_{i, k}, \boldsymbol{x}_{i+1, k}, \boldsymbol{\eta}_{i, k}\right), \\
& \boldsymbol{x}_{n, k+1}=f_{n}\left(\boldsymbol{x}_{n-1, k}, \boldsymbol{x}_{n, k}, Q_{k}^{\text {out }}, v_{k}^{\text {out }}, \boldsymbol{\eta}_{n, k}\right),
\end{aligned}
$$

where $f_{i}$ is specified by the traffic model, $Q_{k}^{i n}$ is the number of vehicles entering segment 1 during the interval $\Delta t_{k}=t_{k+1}-t_{k}$ with average speed $v_{k}^{\text {in }}, Q_{k}^{\text {out }}$ is the outflow leaving a 'fictitious' segment $n+1$, with an average speed $v_{k}^{\text {out }} . \boldsymbol{\eta}_{k}$ is a Gaussian disturbance vector, reflecting random fluctuations and the effect of modelling errors in the state evolution. Note that $Q_{k}^{i n}, v_{k}^{i n}$, and $Q_{k}^{\text {out }}$,

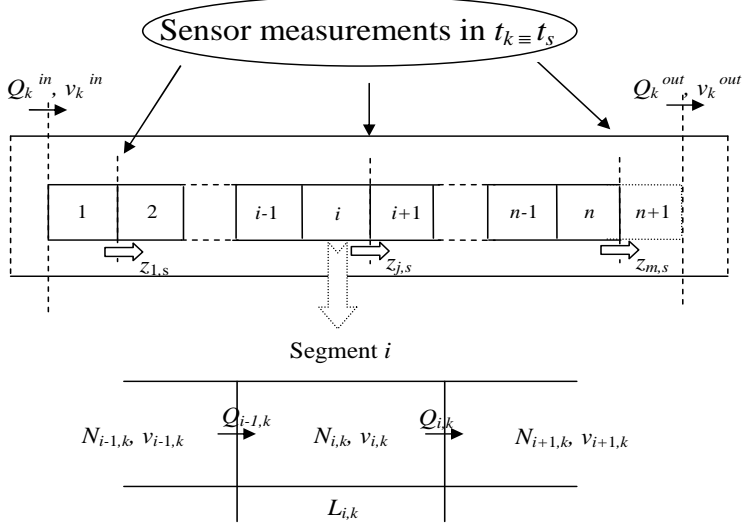

Fig. 1. Freeway segments and measurement points. $Q_{i}$ is the number of vehicles leaving segment $i, N_{i}$ and $v_{i}$ are the average number of vehicles and speed in segment $i$.

$v_{k}^{\text {out }}$ are respectively, inflow and outflow boundary variables. They are not traffic states and are not estimated. They are supplied by the traffic detectors. A chain of interconnected segments is considered, together with their boundary conditions.

In this paper the general state-space description (1)-(3) takes a particular form of the recently developed compositional stochastic macroscopic traffic model (Boel and Mihaylova 2006). This speed-extended cell-transmission model describes the complex traffic behaviour with forward and backward propagation of traffic perturbations and is suitable for large networks and for distributed processing. The forward and backward traffic perturbations were characterised by (Daganzo 1994) through deterministic sending and receiving functions where piecewise affine representations are used. In (Boel and Mihaylova 2006) speeddependent random sending and receiving functions are introduced that represent also the evolution of the average speed in each segment. The model is given in concise form as Algorithm 1.

The sending function $S_{i, k}$ for segment $i$, having length $L_{i}$, is calculated by (4). $S_{i, k}$ represents the vehicles that "intend to leave" segment $i$ within $\Delta t_{k}$. The receiving function $R_{i, k}(6)$ expresses the maximum number of vehicles that are allowed to enter segment $i+1$. In (6) $N_{i+1, k}^{\max }$ characterises the maximum number of vehicles that can simultaneously be present in segment $i+1$ at sample time $t_{k} . N_{i+1, k}^{\max }$ depends on the available space, $L_{i+1}$ time the number of lanes $\ell_{i+1, k}$, in segment $i+1$, on the average length $A_{\ell}$ of vehicles, the average speed $v_{i+1, k}$ and the time distance $t_{d}$ between two vehicles (in order to allow safe driving).

The evolution of $N_{i, k+1}$ is governed by the principle of conservation of vehicles (9). The traffic density $\rho_{i, k+1},[v e h / \mathrm{km} /$ lane $]$, is given by (9). The anticipated density $\rho_{i, k+1}^{a n t i c}$ is then obtained as a weighed average between the density of segment $i$ and segment $i+1$, (11). This corresponds to the 
drivers' tendency usually to look ahead when they change their speed. The average vehicle speed $v_{i, k+1}$ is a function of the 'intermediate' speed $v_{i, k+1}^{\text {interm }}$, calculated in step 5 of Algorithm 1, and of the equilibrium speed satisfying a speed-density relation $v^{e}\left(\rho_{i, k+1}^{a n t i c}\right)$ (Kotsialos et al. 2002).

Design traffic parameters are: the free-flow speed $v_{\text {free }}$, the critical density $\rho_{\text {crit }}$ (density below which the interactions between vehicles will be negligible), the density in jam, $\rho_{\text {jam }}$, above which the vehicles do not move, and the minimum vehicle speed $v_{\text {min }}$. Other details for the model can be found in (Boel and Mihaylova 2006) where this extended cell-transmission model has been validated both against the well established METANET model (Papageorgiou and Blosseville 1989, Kotsialos et al. 2002), and over real traffic data.

Algorithm 1. The compositional traffic model.

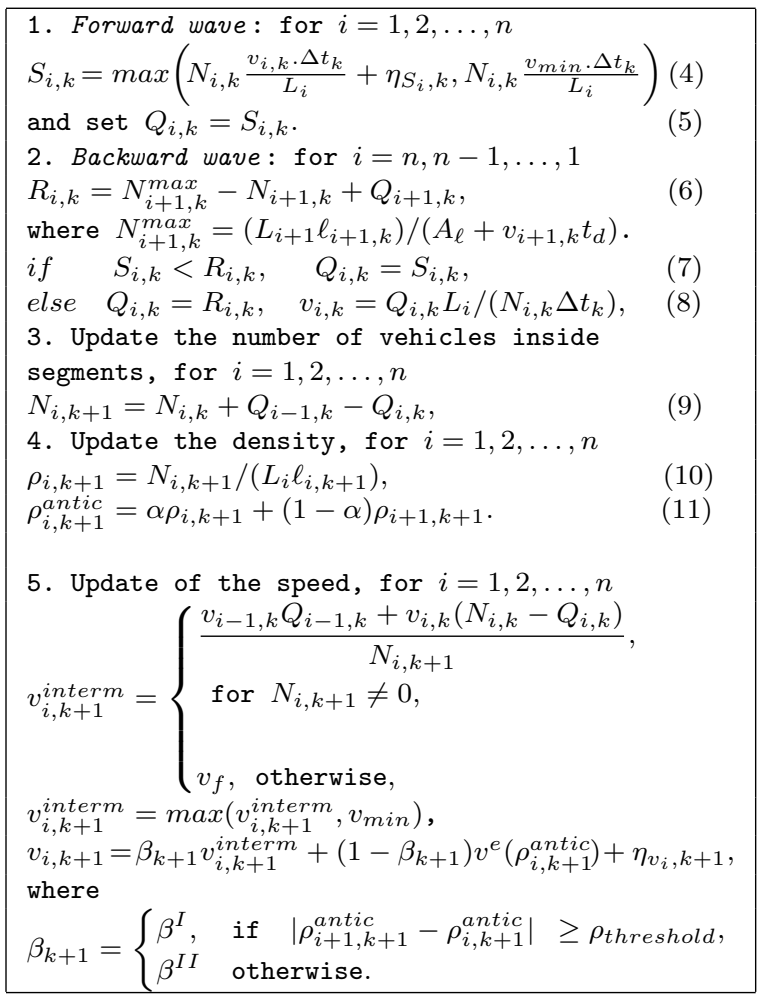

\subsection{Measurement Model}

Sensors (magnetic loops, video cameras, radar detectors) are located at boundaries between some segments. Usually, measurements are collected at the entrance and at the exit of the considered road stretch, at the on-ramps and off-ramps, etc.

Let us consider $m$ sensors along the stretch. Traffic states are measured at discrete time instants. The overall measurement vector $\boldsymbol{z}_{s}=$ $\left(\boldsymbol{z}_{1, s}^{T}, \boldsymbol{z}_{2, s}^{T}, \ldots, \boldsymbol{z}_{m, s}^{T}\right)^{T}$ at time $t_{s}$ consists of local measurement vectors $\boldsymbol{z}_{j, s}=\left(Q_{j, s}, v_{j, s}\right)^{T}$, where $j \in \mathcal{J}=\{1,2, \ldots, m\} . Q_{j, s}$ is the noisy measurement of the number of vehicles crossing the boundaries between the corresponding segment $i$ and segment $i+1$ during the time interval $\Delta t_{s}=t_{s+1}-t_{s}$, and $v_{j, s}$ is the measured mean speed of these vehicles. The intervals $\Delta t_{s}$ are typically several times longer than the intervals $\Delta t_{k}$ between successive state update steps. In case that $\Delta t_{s}>\Delta t_{k}$ we take measurements at each time step.

Consider the measurement equation

$$
\boldsymbol{z}_{s}=\boldsymbol{h}\left(\boldsymbol{x}_{s}, \boldsymbol{\xi}_{s}\right),
$$

where the measurement noise $\boldsymbol{\xi}$ is a white Gaussian process, independent from the system noise $\boldsymbol{\eta}$. If the interval $\Delta t_{s}$ is different from $\Delta t_{k}$ with $q$ steps, i.e. $\Delta t_{s}=q \Delta t_{k}$, the vehicles crossing the boundaries have to correspond to the same time interval as the measurements. Equation (12) takes further the form

$$
\boldsymbol{z}_{j, s}=\left(\begin{array}{c}
\bar{Q}_{j, s} \\
\bar{v}_{j, s}
\end{array}\right)+\boldsymbol{\xi}_{j, s}
$$

where $\bar{Q}_{j, s}$ is the sum of the number of vehicles (calculated by the state model) crossing the boundary between segments $i$ and $i+1$ within the interval $\Delta t_{s}$, and $\bar{v}_{j, s}$ is their average speed.

\section{AN UNSCENTED KALMAN FILTER FOR TRAFFIC FLOW ESTIMATION}

The UKF relies on the unscented transformation (Julier et al. 1995, Julier and Uhlmann 2004, Wan and van der Merwe 2001), a method for calculating the statistics of a random variable which undergoes a nonlinear transformation. Consider propagating a random variable $\boldsymbol{x}$ (with dimension $n_{x}$ ) through a nonlinear transformation $\boldsymbol{y}=\boldsymbol{f}(\boldsymbol{x})$. Assume that $\boldsymbol{x}$ has mean $\hat{\boldsymbol{x}}$ and covariance matrix $\boldsymbol{P}$. To calculate the statistics of $\boldsymbol{y}$, a matrix $\mathcal{X}$ of $2 . n_{x}+1$ sigma points $\mathcal{X}_{i}$ is formed.

The system state update is performed based on these sigma $\mathcal{X}_{i}$ points. To compute the measurement update step, we propagate the sigma points through the measurement function $\boldsymbol{h}$ and we get transformed points $\mathcal{Z}_{i, k / k-1}$ that form the matrix $\boldsymbol{Z}_{k / k-1}$. Similarly to the Kalman filter, the Kalman gain $\boldsymbol{K}$, the state estimate $\hat{\boldsymbol{x}}$ and the corresponding covariance matrix $\boldsymbol{P}$ are updated by (14)-(16). The UKF equations are given as Algorithm 2. We implemented the UKF using an augmented state vector concatenating the original state and the noise variables: $\boldsymbol{x}_{k}^{a}=$ $\left(\boldsymbol{x}_{k}^{T}, \boldsymbol{\eta}_{k}^{T}, \boldsymbol{\xi}_{k}^{T}\right)^{T}$ (Wan and van der Merwe 2001). The corresponding matrix with sigma points is $\mathcal{X}^{a}=\left(\left(\mathcal{X}^{x}\right)^{T},\left(\mathcal{X}^{\eta}\right)^{T},\left(\mathcal{X}^{\xi}\right)^{T}\right)^{T}$. The sigma points of the UKF are deterministically chosen so that they exhibit certain properties, e.g. have a given mean and covariance. The UKF is formulated for Gaussian distributions of the noises. 
Algorithm 2. The Unscented Kalman Filter.

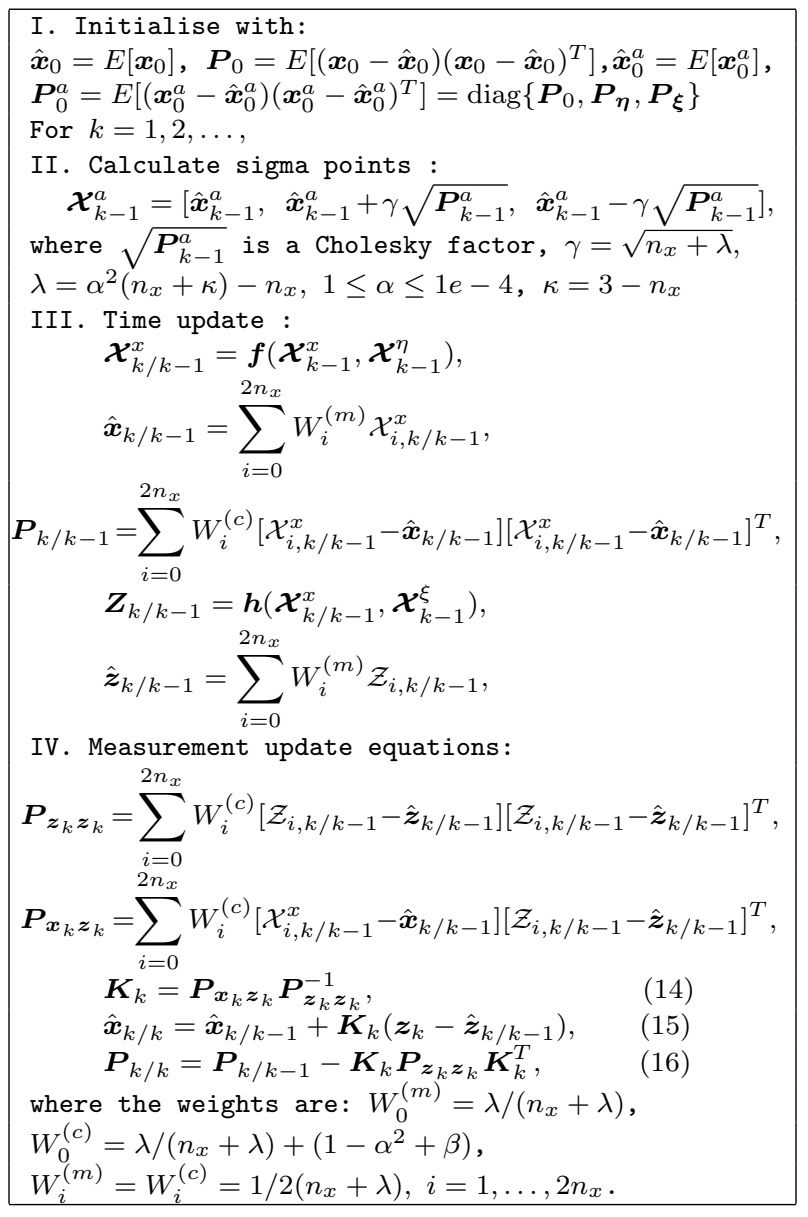

\section{UKF PERFORMANCE EVALUATION}

\subsection{Investigations with Synthetic Data}

The UKF performance is evaluated versus the PF developed in (Mihaylova and Boel 2004) over of freeway stretch of $4[\mathrm{~km}]$ consisting of eight segments with data, having periods of congestion. The data are generated by the compositional model (Boel and Mihaylova 2006) with independent measurement noises for different runs and with different initial state conditions. The congestion is due to variations in the inflow $Q_{k}^{i n}$ and outflow $Q_{k}^{\text {out }}$ (shown in Fig. 2) within the period $1.12 \mathrm{~h}-1.7 \mathrm{~h}$ and due to the fall in the speed $v_{k}^{\text {out }}$ within the interval $2.4 \mathrm{~h}-2.65 \mathrm{~h}$. The measurements are generated, by adding measurement noises to the counted number of vehicles $Q_{i, k}$ and to the speed $v_{i, k}$ for segments 1 and 8. These measurements are used in the filters also as inflow/ outflow boundary conditions (for the state model). The augmented state vector is $\boldsymbol{x}_{k}=\left(\boldsymbol{x}_{1, k}^{T}, \boldsymbol{x}_{2, k}^{T}, \ldots, \boldsymbol{x}_{8, k}^{T}\right)^{T}$, i.e. $i=1,2, \ldots, 8$, and the measurement vector is $\boldsymbol{z}_{s}=\left(\boldsymbol{z}_{1, s}^{T}, \boldsymbol{z}_{8, s}^{T}\right)^{T}$.

The per minute aggregated measurements are supplied to the UKF and PF as would be the case with real data. The state prediction is performed also at each intermediate state update time step.
The states of all segments between two measurements are estimated as one augmented state vector. The filters' performance is evaluated by the
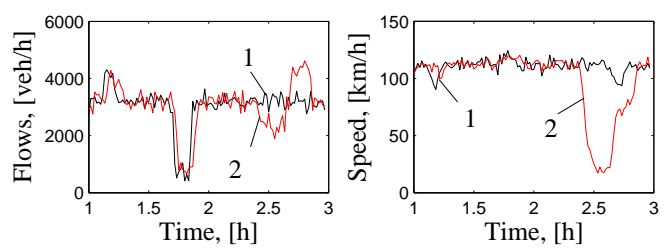

Fig. 2. Boundary conditions: 1 - in, 2 - out

Root mean square errors (RMSEs) $\epsilon\left(\hat{x}_{i, k}\right)=$ $\left[\frac{1}{r} \sum_{i=1}^{r}\left(\varepsilon_{i, k}\right)^{T}\left(\varepsilon_{i, k}\right)\right]^{1 / 2}$, where $\hat{x}_{i, k}, \varepsilon_{i, k}=x_{i, k}-$ $\hat{x}_{i, k}$ are the errors between the actual $x_{i, k}$ and estimated states over $r$ independent Monte Carlo runs. Table 3 gives the parameters of the state model. The evolution of the flow and speed in time (for one realisation) is shown in Figs. 3 and 4. We see the backward wave on the evolution of the speed and flow in time. The flow-density and the speed-flow diagrams have the typical bell-shaped forms. The filters' performance is evaluated for $r=100$ independent Monte Carlo runs. RMSEs for all the eight segments, with respect to density, speed and flow, are presented in Figs. 5-6.
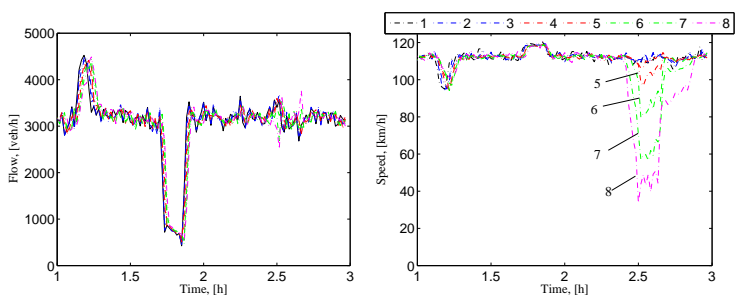

Fig. 3. Diagrams based on the PF and UKF estimates
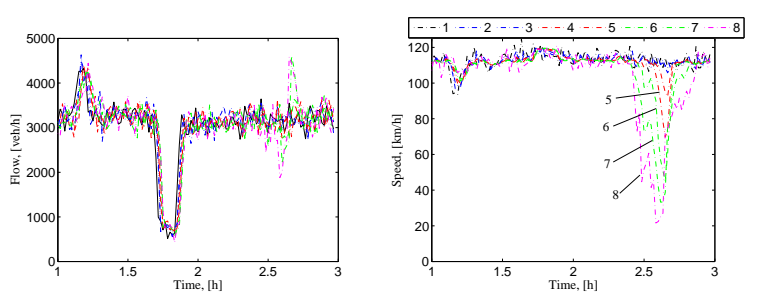

Fig. 4. Diagrams based on the PF and UKF estimates

We see the influence of the backward wave on these RMSEs. We observe that the RMSE values in segment 1 are smaller than their values in the intermediate segment 5 (it is also due to the fact that there are no sensor data in this segment). According to these results the $\mathrm{PF}$ estimates are more accurate than the UKF estimates. However, the $\mathrm{PF}$ complexity is more computationally expensive than the UKF. The complexity of the PF is proportional to the the number of particles times the dimension of the overall state vector, $M . n_{x}$, whilst the complexity of the UKF is proportional to the number $2 . n_{x}+1$ of sigma points. Note that $n_{x}$ is equal to the number of segments $n$ times the number of states 2 in a segment. We calculated the ratio between the PF and UKF computational 
time and it is: 2.8 (with $M=100$ particles), 5.45 (with $M=200$ ), 15 (with $M=500$ ). In general, the number of necessary particles is increasing with the increased number of states for reaching a certain accuracy, but not very much. It is difficult to characterise in general the PF accuracy and complexity because they highly depend on the road structure and the traffic conditions.

Table 3. Parameters of the UKF and PF

\begin{tabular}{|c|}
\hline$v_{\text {free }}=120[\mathrm{~km} / \mathrm{h}], v_{\text {min }}=7.4[\mathrm{~km} / \mathrm{h}]$ \\
\hline$\rho_{\text {crit }}=20.89[\mathrm{veh} / \mathrm{km} / \mathrm{lane}], \rho_{\text {jam }}=180[\mathrm{veh} / \mathrm{km}]$ \\
\hline$\alpha=0.65, \beta_{k+1}= \begin{cases}0.25, & \text { if }\left|\rho_{i+1, k+1}^{\text {antic }}-\rho_{i, k+1}\right| \geq 2, \\
0.75, & \text { otherwise. }\end{cases}$ \\
\hline$\Delta t_{i}=10[\mathrm{sec}], t_{d}=2[\mathrm{sec}], L_{i}=0.5[\mathrm{~km}], i=1, \ldots, 8$, \\
\hline$M=200$ particles, $t_{d}=2[\mathrm{sec}], A_{\ell}=0.01[\mathrm{~km}], \ell_{i}=3$ \\
\hline $\operatorname{cov}\left\{\eta_{S_{i, k}}\right\}=\left(0.03 N_{i, k} v_{i, k} \Delta t_{k} / L_{i}\right)^{2}[\mathrm{veh}]^{2}$ \\
\hline $\operatorname{cov}\left\{\eta_{Q_{i}}\right\}=1^{2}[\mathrm{veh}]^{2}, \operatorname{cov}\left\{\eta_{v_{i}}\right\}=3.5^{2}[\mathrm{~km} / \mathrm{h}]^{2}$ \\
\hline $\operatorname{cov}\left\{\xi_{Q_{i}}\right\}=1^{2}[\mathrm{veh}]^{2}, \operatorname{cov}\left\{\xi_{v_{i}}\right\}=5^{2}[\mathrm{~km} / \mathrm{h}]^{2}$ \\
\hline
\end{tabular}

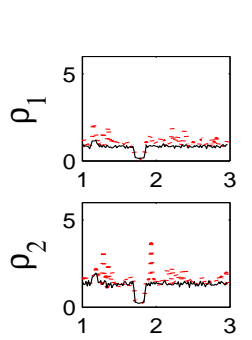

Root Mean Square Errors
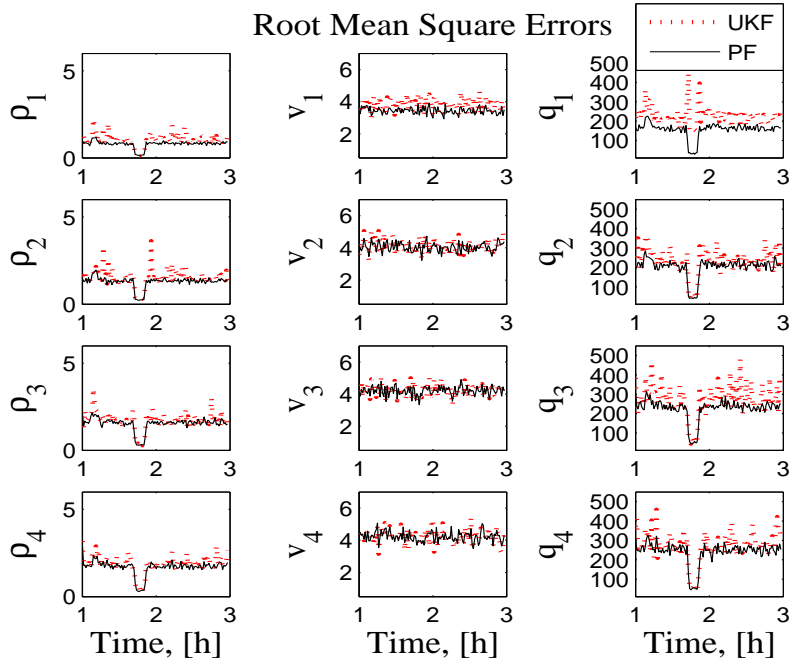

Time, $[\mathrm{h}]$

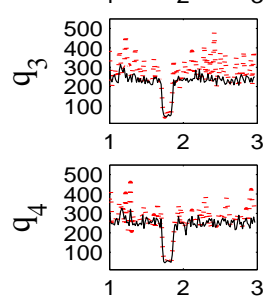

Time, [h]

Fig. 5. UKF and PF RMSEs of the density (for all lanes) $[v e h / k m]$, speed $[\mathrm{km} / \mathrm{h}]$ and flow $[v e h / h]$ of segments $1-4$

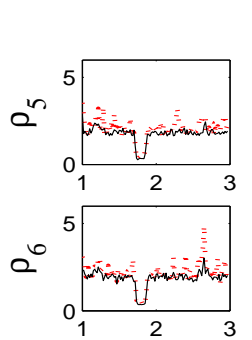

Root Mean Square Errors
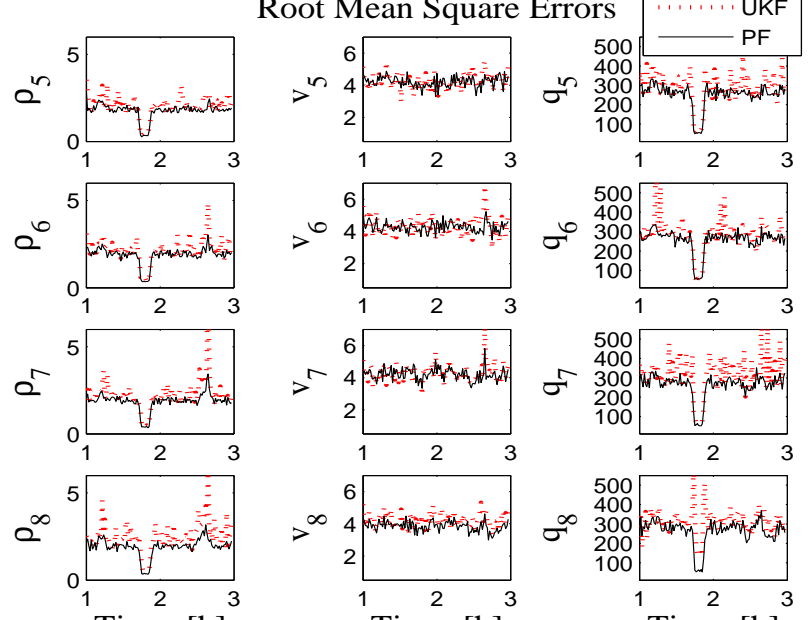

Time, $[\mathrm{h}]$

Time, $[\mathrm{h}]$

Fig. 6. UKF and PF RMSEs of the density (for all lanes) $[v e h / k m]$, speed $[\mathrm{km} / \mathrm{h}]$ and flow $[v e h / h]$ of segments $5-8$

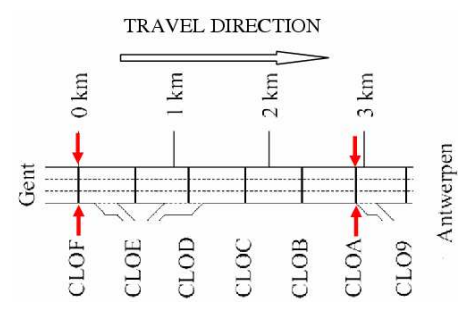

Fig. 7. Schematic representation of the segmentation of the E17 case study freeway. The labels CLOF to CLO9 indicate the locations of the traffic measurement cameras. The vertical arrows indicate the location of the used measurements.

\subsection{Application of the UKF to Real Traffic Data}

The UKF and PF performance has also been evaluated with real data, over a stretch of E17 (between CLOF and CLOA on Fig. 7) freeway between the cities of Ghent and Antwerp. E17 is one of the very important Belgian freeways subject to frequent congestion. Measurement data are available from video cameras installed at location CLOA, CLOB, CLOD, CLOE, and CLOF, including the total number of vehicles that cross the sensor location during each one minute interval, and the average speed of these vehicles during that one minute interval. We tested the PF and UKF using data measured from September, 2001 from 6.4 [h] a.m. till 10.6 [h] a.m., which period includes heavy congestion. The data are supplied from two sensors installed at CLOF and CLOA (Fig. 7). The link CLOF to CLOA contains an offramp towards and an on-ramp from a parking lot, but we assume that the flow of vehicles using this parking lot is negligible so that the conservation equation (9) remains valid in the state prediction step. The parameters of the models and of the filters are given in Table 4 . The filters generate estimates of the state of each segment in a link, and also of the speed and density (and hence also of the flow) at each boundary between segments. Figures 8 presents flow-density diagrams plotted based on the estimates. The bell-shaped diagram shows nicely that the estimated states indeed have properties as one can expect for traffic data. These estimates of the density, speed, and flow at the boundaries are compared with the measured data in the intermediate segment boundary CLOD (Figs. 9 and 10).

Table 4. Parameters of the UKF and PF

\begin{tabular}{|c|}
\hline$v_{\text {free }}=120[\mathrm{~km} / \mathrm{h}], \quad v_{\min }=7.4[\mathrm{~km} / \mathrm{h}]$ \\
\hline$\beta_{k+1}= \begin{cases}0.3, \quad \text { if }\left|\rho_{i+1, k+1}^{\text {antic }}-\rho_{i, k+1}\right| \geq 2, \\
0.7, \quad \text { otherwise. }\end{cases}$ \\
\hline$L_{1}=L_{2}=L_{3}=0.6[\mathrm{~km}], L_{4}=L_{5}=0.5[\mathrm{~km}]$ \\
\hline$\Delta t_{i}=10[\mathrm{sec}], t_{d}=1.5[\mathrm{sec}], A_{\ell}=0.01[\mathrm{~km}]$ \\
\hline$\rho_{\text {crit }}=20.89[\mathrm{veh} / \mathrm{km} /$ lane $], \quad \rho_{\text {jam }}=180[\mathrm{veh} / \mathrm{km}]$ \\
\hline$M=100$ particles, $\alpha=0.65$ \\
\hline Gaussian noises $\eta_{S_{i, k}, \eta_{v_{i, k}} \text { with covariances: }}$ \\
\hline $\operatorname{cov}\left\{\eta_{S_{i, k}}\right\}=\left(0.035 N_{i, k} v_{i, k} \Delta t_{k} / L_{i}\right)^{2}[\mathrm{veh}]^{2}$ \\
\hline $\operatorname{cov}\left\{\eta_{v_{i}}\right\}=3.5^{2}[\mathrm{~km} / \mathrm{h}]^{2}, \operatorname{cov}\left\{\eta_{Q_{i}}\right\}=1^{2}[\mathrm{veh}]^{2}$, \\
\hline $\operatorname{cov}\left\{\xi_{v_{i}}\right\}=5^{2}[\mathrm{~km} / \mathrm{h}]^{2}, \operatorname{cov}\left\{\xi_{Q_{i}}\right\}=1^{2}[\mathrm{veh}]^{2}$ \\
\hline
\end{tabular}



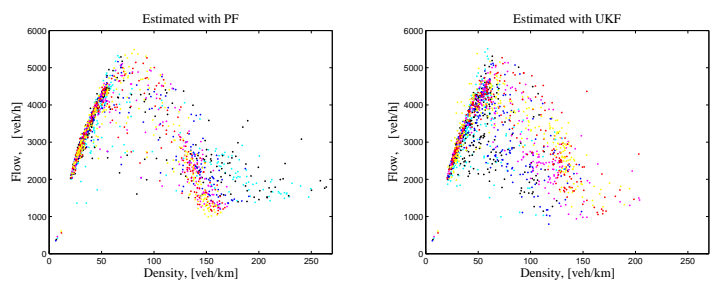

Fig. 8. Diagrams based on the PF and UKF estimated states
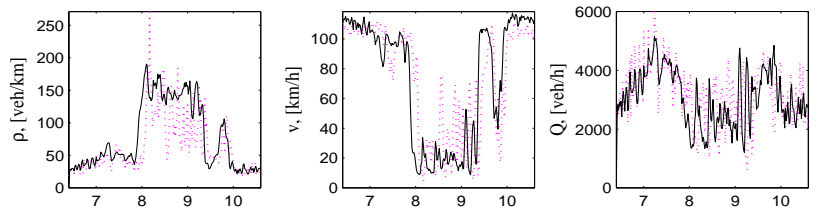

Fig. 9. PF estimated states (solid line) versus measured states in CLOD (dashed line)
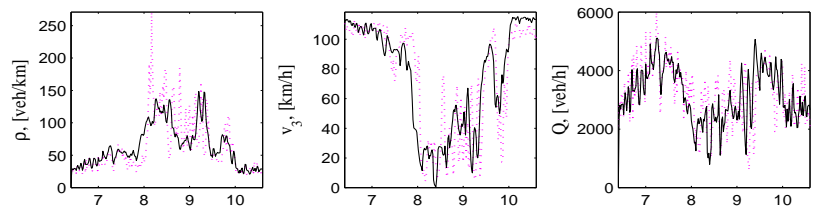

Fig. 10. UKF estimated states (solid line) versus measured states in CLOD (dashed line)

\section{CONCLUSIONS AND OPEN ISSUES}

This paper presents an Unscented Kalman filter for the freeway traffic flow estimation and compares its performance with respect to a particle filter. The Unscented Kalman filtering is a promising method for traffic flow estimation, requiring small computational costs. The UKF is developed using traffic and observation models with aggregated variables. The traffic is modelled by a recently developed stochastic compositional traffic model with interconnected states of neighbour segments. The UKF and PF performance is investigated and validated by simulated data and by real traffic data from a Belgian freeway. An advantage of the UKF compared to the $\mathrm{PF}$ is that it is less computationally expensive. Both the results with simulated and real traffic data confirm that the UKF provides accurate tracking performance, however, slightly less accurate than the PF. Both the UKF and the PF are suitable methods for real-time traffic estimation, and both are easy to implement because of the fact that they do not require linearisation. Both the UKF and PF, extended with on-line mode detection logic, can be used for on-line traffic control strategies, e.g. within the model predictive control framework.

Acknowledgments. Financial support by the project DWTC-CP/40 "Sustainability effects of traffic management", sponsored by the Belgian government is acknowledged, and by the Belgian
Programme on Inter-University Poles of Attraction initiated by the Belgian State, Prime Minister's Office for Science, Technology and Culture. We also thank the "Vlaams Verkeerscentrum Antwerpen", Antwerp, Belgium for providing the data used in this study.

\section{REFERENCES}

Boel, R. and L. Mihaylova (2006). A compositional stochastic model for real-time freeway traffic simulation. Transportation Research $B$ 40(4), 319-334.

Chen, R. and J. Liu (2000). Mixture Kalman filters. J. Royal Statist. Soc. B 62, 493-508.

Daganzo, C. (1994). The cell transmission model: A dynamic representation of highway traffic consistent with the hydrodynamic theory. Transp. Res. B 28B(4), 269-287.

Helbing, D. (2002). Traffic and related self-driven many-particle systems. Rev. of Modern Phys. 73, 1067-1141.

Hoogendoorn, S.P. and P.H.L. Bovy (2001). Stateof-the-art of vehicular traffic flow modelling. J. of Systems Contr. Engineer - Proc. of the Institution of Mech. Engineers, Part I 215(14), 283-303.

Julier, S. and J. Uhlmann (2004). Unscented filtering and nonlinear estimation. Proceedings of the IEEE 92(3), 401-422.

Julier, S., J. Uhlmann and H. Durrant-White (1995). A new approach for filtering nonlinear systems. In: Proc. of the American Control Conf.. Washington, DC. pp. 1628-1632.

Kotsialos, A., M. Papageorgiou, C. Diakaki, Y. Pavlis and F. Middelham (2002). Traffic flow modeling of large-scale motorway using the macroscopic modeling tool METANET. IEEE Transactions on Intelligent Transportation Systems 3(4), 282-292.

Mihaylova, L. and R. Boel (2004). A particle filter for freeway traffic estimation. In: Proc. 43rd IEEE Conf. on Dec. \& Contr.. pp. 2106-2111.

Papageorgiou, M. and J.-M. Blosseville (1989). Macroscopic modelling of traffic flow on the boulevard Périphérique in Paris. Transportation Research B 23B(1), 29-47.

Sun, X., L. Muñoz and R. Horowitz (2003). Highway traffic state estimation using improved mixture Kalman filters for effective ramp metering control. In: Proc. 42th IEEE Conf. on Dec. and Contr.. USA. pp. 6333-6338.

Wan, E. and R. van der Merwe (2001). Kalman Filtering and Neural Networks. Chapter 7. The Unscented Kalman Filter 7, pp. 221-280. Wiley Publishing.

Wang, Y. and M. Papageorgiou (2005). Realtime freeway traffic state estimation based on extended Kalman filter: a general approach. Transportation Research B 39B(2), 141-167. 